\title{
Distribuição espacial da mortalidade por tuberculose em Salvador, Bahia, Brasil
}

\author{
Spatial distribution of tuberculosis \\ mortality in Salvador, Bahia, Brazil
}

Fábio Frias Mota 1

Ligia Maria Vieira-da-Silva 1

Jairnilson Silva Paim 1

Maria da Conceição Nascimento Costa 1

\footnotetext{
1 Instituto de Saúde Coletiva, Universidade Federal da Bahia. Rua Padre Feijó 29, Salvador BA 40110-170, Brasil. ligiamvs@ufba.br jairnil@ufba.br mcosta@ufba.br
}

\begin{abstract}
An ecological study was conducted to describe the urban spatial distribution of mortality attributable to tuberculosis in Salvador, Bahia, Brazil in 1991, 1994, and 1997. The unit of analysis was the Health District (HD). The HD with the highest standardized rates was Suburbio Ferroviário in 1991, 1994, and 1997, with 15.7, 10.6, and 10.6/100.000 inhabitants, respectively. Inequalities in mortality between HDs were high. The year 1997 showed the lowest standardized rates. The proportional mortality ratios for tuberculosis when compared with total deaths from infectious diseases were 20.7\%, 18.2\%, and 16.9\% for 1991, 1994, and 1997 respectively. The highest rates were observed in the age group over 65 years. The authors argue the possibilities of using the results for the implementation of equitable local health policies and health surveillance measures, since the methodology identified both the HD with the highest risk and the most heavily affected age groups.
\end{abstract}

Key words Tuberculosis; Mortality; Spatial Distribution

Resumo Com o objetivo de descrever a distribuição espacial da mortalidade por tuberculose em Salvador nos anos de 1991, 1994 e 1997, foi realizado um estudo ecológico, descritivo, tendo como unidade de análise o Distrito Sanitário (DS). Foram calculados os coeficientes padronizados de mortalidade por tuberculose e a mortalidade proporcional por tuberculose, em relação ao conjunto das doenças infecciosas e parasitárias, utilizando-se as declarações de óbitos como fonte de dados. O DS com os maiores coeficientes padronizados foi o Subúrbio Ferroviário, com taxas de 15,7, 10,6 e 10,6/100 mil habitantes, em 1991, 1994 e 1997, respectivamente. Em 1997, foram observadas as menores taxas padronizadas. A mortalidade proporcional por tuberculose em Salvador, nos anos de 1991, 1994 e 1997, representou 20,7\%, 18,2 \% e 16,9\%, respectivamente, do total das mortes por doenças infecciosas e parasitárias. A faixa etária de maior risco, nos anos estudados, foi aquela acima de 65 anos. Os autores discutem as possibilidades de utilização dos resultados deste estudo para a implementação de políticas locais de saúde equânimes e desenvolvimento de operações de vigilância da saúde, tendo em vista a identificação de DS com maior risco de óbito e as faixas etárias mais acometidas.

Palavras-chave Tuberculose; Mortalidade; Distribuição Espacial 


\section{Introdução}

A persistência da tuberculose em escala internacional, a despeito da existência de estratégias terapêuticas eficazes, revela não apenas a diversidade de fatores envolvidos na sua determinação mas sobretudo a complexidade do seu controle (Hopewell, 2002; WHO, 1999).

A magnitude do problema no início da década de 90, se expressava em estimativas de ocorrência de cerca de 7,5 milhões de casos e uma taxa de notificação de 74,6/100 mil habitantes (Raviglione et al., 1996). Também a mortalidade mundial estimada era de 48/100 mil habitantes em 1990, sendo que $98 \%$ desses casos teriam ocorrido nos países subdesenvolvidos (Raviglione et al., 1996). Em resposta a essa situação, a Organização Mundial da Saúde (OMS) considerou a tuberculose como uma emergência global em 1993 e formulou a estratégia que ficou conhecida como DOTS (Directly Observed Treatment, Short-course), que combina o tratamento de curta duração com medidas políticas, organizacionais e de vigilância (WHO, 1999).

Contudo, apesar dos progressos registrados e da eficácia da estratégia adotada, dos 179 países que notificaram casos de tuberculose, apenas $46 \%$ estavam implementando a estratégia DOTS (WHO, 2002) e somente $27 \%$ dos casos notificados estavam sendo submetidos ao DOTS (Dye et al., 2002). Por esses motivos, em 2000 a Assembléia da OMS convocou diversas instituições governamentais e não governamentais para participar da ação denominada Parceria Global para Parar a Tuberculose (Lee et al., 2002).

No Brasil, a mortalidade por tuberculose começou a cair abruptamente a partir da década de 50 com o advento da quimioterapia, tendo-se verificado a redução da velocidade de decréscimo nas décadas seguintes. Nas capitais brasileiras este decréscimo foi de $61,4 \%$ entre 1970-1979, havendo um declínio médio de 10\% ao ano, sempre com coeficientes de mortalidade mais altos nas regiões Norte e Nordeste (Lima, 1983). Entre 1977 e 1987, o porcentual de redução foi de $51,7 \%$ ou seja, em média $5,4 \%$ ao ano (Hijjar, 1992). Já a taxa de incidência sofreu redução de 63,4/100 mil habitantes em 1981 para 48,2/100 mil habitantes em 1990 (Hijjar, 1992), mantendo-se nesse patamar $(48,0 / 100$ mil habitantes) em 1999 (Ruffino-Netto, 2002).

Embora o tratamento de curta duração (seis meses) tenha sido adotado desde 1979 e a supervisão do mesmo tivesse ocorrido localizadamente (Ruffino-Netto, 2002), apenas em 1998, com o Plano Nacional de Controle da Tuberculose, é que a OMS considera que o país tenha aderido à estratégia DOTS (WHO, 2002). Desde então foi incluído entre os 22 países considerados como de alta incidência, com uma taxa estimada por aquela instituição de 68/100 mil habitantes ao ano em 2000, sendo as coberturas estimadas inferiores a 7\% (WHO, 2002). Chama a atenção, no relatório da OMS de 2002, a proporção de casos notificados e não avaliados no Brasil, em torno de $88 \%$ do total (WHO, 2002). Esse problema levou o Ministério da Saúde (MS), em 2001, a deflagrar um processo de avaliação da situação de controle da tuberculose no país com ênfase no aperfeiçoamento do Sistema de Informação. Um melhor acompanhamento acerca da ocorrência, evolução e mortalidade da tuberculose no nosso meio requer não apenas o aperfeiçoamento do sistema específico de informação, mas impõe também a realização de investigações descritivas a esse respeito.

Em Salvador, existem alguns estudos que analisaram a distribuição espacial dos casos notificados de tuberculose, as condições de vida dos doentes e a recidiva da doença, na década de 80 (Carneiro \& Mota, 1986; Lima, 1983; Menezes, 1986). Naquele período, a incidência por tuberculose era de 125/100 mil habitantes, superior portanto à média nacional sendo elevada a taxa de abandono, em torno de 19\% (Carneiro \& Mota, 1986). A incidência era maior na faixa etária entre 20 e 40 anos e em ocupações sem qualificação com elevado porcentual de hospitalizações (Menezes, 1986). O trabalho de Lima evidenciou ainda maior incidência da tuberculose nos bairros de menor renda, de maior concentração de favelas e de maior densidade populacional (Lima, 1983).

Não existem estudos sobre a mortalidade por esta causa na década de 90 para o referido município, nem pesquisas publicadas sobre diferenciais intra-urbanos. Os trabalhos sobre mortalidade em escala nacional e regional têm sido relevados a um plano secundário, tendo em vista o subregistro de dados em alguns estados da federação. Contudo, em se tratando de capitais, essa informação tem maior validade e sua descrição e análise pode não apenas suprir essa lacuna como contribuir para com o conhecimento acerca da evolução da tuberculose no nosso meio. Por esses motivos, o presente estudo pretende descrever a distribuição espacial da mortalidade por esse agravo em Salvador, nos anos de 1991, 1994 e 1997.

\section{Metodologia}

Foi realizado um estudo descritivo do tipo ecológico da mortalidade por tuberculose em Salvador, nos anos de 1991, 1994 e 1997, em que a 
unidade de análise foi o Distrito Sanitário (DS). Este município possui 12 DS que correspondem a menor unidade técnica-administrativa do sistema municipal de saúde, cuja constituição é compatível com as 75 Zonas de Informação (ZI), áreas em que a cidade foi dividida pela Companhia de Desenvolvimento da Região Metropolitana de Salvador (CONDER), de acordo com critérios físico-urbanísticos, administrativos, de planejamento e de compatibilização com os setores censitários da Fundação Instituto Brasileiro de Geografia e Estatística (IBGE).

Como fonte de dados foram utilizadas fotocópias das declarações de óbitos (DO) dos residentes em Salvador e falecidos nos referidos anos, que se encontravam arquivadas na Secretaria de Saúde do Estado da Bahia (SESAB). Foram considerados todos os óbitos que apresentavam como causa básica a tuberculose (CID9 010-18.9) ou agravos tardios relacionados à tuberculose (CID9 137.1-137.4).

Para cada DS estimaram-se os Coeficientes de Mortalidade por tuberculose, global e por faixa etária, bem como a Mortalidade Proporcional em relação às Doenças Infecciosas e Parasitárias (DIP). Como denominador dos coeficientes utilizaram-se dados populacionais do Censo Demográfico de 1991, fornecidos pelo IBGE e estimativas para os demais anos. Os coeficientes foram padronizados por idade, pelo método indireto (Rothman, 1986), considerando-se as taxas de mortalidade por esta causa na população de Salvador, para o ano de 1991, como padrão.

Os diferenciais intra-urbanos foram analisados segundo o cálculo da razão de mortalidade, aqui denominada de razão de desigualdade, tomando-se como referência o menor coeficiente de mortalidade entre os DS.

Foram descartadas algumas DO para as quais, no campo relativo ao "endereço habitual" havia referência a outro local de residência (em geral, municípios da região metropolitana), e no campo "município de residência" encontrava-se registrado o Município de Salvador. Entretanto, estes óbitos foram considerados como de residentes no município, pelo órgão responsável pelas estatísticas de mortalidade em Salvador, visto que apenas o nome do município encontra-se registrado no campo específico. Por esse motivo, e por não ter sido possível localizar geograficamente por DS, 2,3\%, 3,0\% e 14\% dos óbitos por tuberculose, respectivamente, nos três anos estudados, existem diferenças entre os totais de óbito do presente estudo e as estatísticas oficiais do MS.

\section{Resultados}

O coeficiente de mortalidade por tuberculose em Salvador foi de 8,9/100 mil habitantes em 1991, 7,6/100 mil habitantes em 1994 e de 7,7/100 mil habitantes em 1997. Os maiores riscos de morte por essa doença foram observados no DS do Subúrbio Ferroviário: 15,7, 10,6 e 9,9/100 mil habitantes, nesta ordem, em cada um dos três anos do estudo. Na maioria dos DS houve, em 1994, uma redução percentual dos coeficientes padronizados (CP), exceto nos DS de Itapagipe, Boca do Rio e Barra/Rio Vermelho/Pituba que apresentaram crescimentos percentuais de 59,3, 26,3 e 68,2, respectivamente. Em 1997, apenas os DS do Centro Histórico e de Pau da Lima apresentaram um crescimento destes coeficientes, quando comparados ao ano de 1994. A razão de desigualdade da mortalidade por essa causa variou entre 1,0 e 4,1 para 1991 e de 1,1 a 3,0 em 1994 (Tabela 1).

Em cada um dos três anos analisados, a mortalidade por tuberculose representou $20,7 \%$, $18,2 \%$ e $16,9 \%$, do total das mortes por DIP. Os maiores percentuais desta relação foram encontrados nos DS Centro Histórico (31,9\%) e São Caetano/Valéria (27,6\%) em 1991; Barra/Rio Vermelho/Pituba (29,3\%) e Liberdade $(22,8 \%)$ em 1994 e Subúrbio Ferroviário (28,0\%); Itapagipe $(19,4 \%)$ e Pau da Lima (21,8\%) em 1997. Este último foi o DS que apresentou, em 1991 e em 1994, o menor percentual, posição que foi ocupada em 1997 pelo DS da Liberdade (Tabela 2). As maiores variações deste indicador no período 1991-1997 foram observadas nos DS Pau da Lima (+69,0\%) e Centro Histórico (-55,2\%) e em 1991-1994 Barra/Rio Vermelho/Pituba (99,3\%) e Pau da Lima (-56,6\%). Chama a atenção a acentuada variação $(+289,3 \%)$ apresentada por este último DS no período 1994-1997.

Observou-se um crescimento constante no risco de morte por tuberculose a partir do grupo etário de 30-39 anos, atingindo o seu ápice na faixa etária acima de 65 anos. Nesta faixa etária e no grupo de 40-49 anos houve uma redução desta mortalidade (Figura 1).

\section{Discussão}

A mortalidade por tuberculose ainda representa um problema de saúde relevante em Salvador e na maioria dos seus DS. Os riscos de morte por esta causa em Salvador nos anos do estudo, representaram cerca do dobro daqueles verificados no Brasil no mesmo período, sendo que para alguns distritos essa relação é de cinco vezes (Ruffino-Netto \& Souza, 1999). 
Tabela 1

Coeficientes bruto e padronizado de mortalidade por tuberculose (por 100 mil habitantes), razão de desigualdade e variação anual segundo Distrito Sanitário de residência. Salvador, Bahia, Brasil, 1991, 1994 e 1997.

\begin{tabular}{|c|c|c|c|c|c|c|c|c|c|c|c|c|c|c|c|}
\hline \multirow[t]{2}{*}{ Distrito Sanitário } & \multicolumn{6}{|c|}{ Coeficiente bruto } & \multicolumn{3}{|c|}{$\begin{array}{l}\text { Coeficiente } \\
\text { padronizado }\end{array}$} & \multicolumn{3}{|c|}{$\begin{array}{c}\text { Razão de } \\
\text { desigualdade }\end{array}$} & \multicolumn{3}{|c|}{ Variação anual (\%) } \\
\hline & 1991 & IC95\% & 1994 & IC95\% & 1997 & IC95\% & 1991 & 1994 & 1997 & 1991 & 1994 & 1997 & $\begin{array}{l}1991- \\
1994\end{array}$ & $\begin{array}{l}1994- \\
1997\end{array}$ & $\begin{array}{l}1991- \\
1997\end{array}$ \\
\hline I - Centro Histórico & 18,9 & $9,4-28,5$ & 5,9 & $0,0-11,1$ & 12,6 & $4,4-20,8$ & 13,4 & 4,1 & 9,9 & 3,5 & 1,2 & 2,8 & $-69,4$ & 141,5 & $-26,1$ \\
\hline II - Itapagipe & 6,7 & $2,6-10,9$ & 10,7 & $5,6-15,8$ & 9,3 & $4,7-15,1$ & 5,9 & 9,4 & 8,1 & 1,6 & 2,7 & 2,5 & 59,3 & $-13,8$ & 37,3 \\
\hline III - São Caetano/Valéria & 10,8 & $6,2-15,4$ & 9,6 & $5,4-13,8$ & 3,1 & $1,6-6,0$ & 11,7 & 10,5 & 2,9 & 3,1 & 3,0 & 1,0 & $-10,3$ & $-72,4$ & $-75,2$ \\
\hline IV - Liberdade & 11,0 & $6,2-15,9$ & 9,3 & $5,0-13,6$ & 5,1 & $3,4-11,3$ & 10,1 & 8,5 & 4,5 & 2,7 & 2,4 & 1,8 & $-15,8$ & $-47,1$ & $-55,4$ \\
\hline V - Brotas & 8,5 & $3,8-12,1$ & 4,8 & $1,7-7,9$ & 3,9 & $1,4-7,6$ & 7,2 & 4,3 & 3,4 & 1,9 & 1,2 & 1,1 & $-40,3$ & $-20,9$ & $-52,8$ \\
\hline $\begin{array}{l}\text { VI - Barra/Rio Vermelho/ } \\
\text { Pituba }\end{array}$ & 5,1 & $2,6-7,6$ & 8,6 & $5,5-11,8$ & 6,8 & $4,0-9,6$ & 4,4 & 7,4 & 5,6 & 1,2 & 2,1 & 1,6 & 68,2 & $-24,3$ & 27,3 \\
\hline VII - Boca do Rio & 3,7 & $0,0-7,9$ & 4,6 & $0,0-9,1$ & 6,7 & $1,3-12,0$ & 3,8 & 4,8 & 5,9 & 1,0 & 1,4 & 1,7 & 26,3 & 22,9 & 55,3 \\
\hline VIII - Itapuã & 7,6 & $2,9-12,3$ & 7,1 & $2,7-11,5$ & 4,0 & $0,8-7,1$ & 8,6 & 8,0 & 3,8 & 2,3 & 2,3 & 1,1 & $-8,0$ & $-52,5$ & $-55,8$ \\
\hline IX - Cabula & 8,4 & $4,9-12,0$ & 6,5 & $3,5-9,5$ & 4,6 & $2,1-7,1$ & 9,8 & 7,5 & 4,4 & 2,6 & 2,1 & 1,3 & $-23,5$ & $-41,3$ & $-55,1$ \\
\hline X - Pau da Lima & 6,3 & $2,2-10,4$ & 3,3 & $0,0-6,1$ & 6,8 & $2,9-10,5$ & 7,4 & 3,8 & 6,4 & 1,9 & 1,1 & 1,8 & $-48,6$ & 68,4 & $-13,5$ \\
\hline XI - Subúrbio Ferroviário & 13,7 & $9,2-18,1$ & 9,2 & $5,7-12,8$ & 10,2 & $7,0-14,8$ & 15,7 & 10,6 & 9,9 & 4,1 & 3,0 & 3,0 & $-32,5$ & $-6,6$ & $-36,9$ \\
\hline XII - Cajazeiras & 4,0 & $0,0-9,4$ & 2,8 & $0,0-6,0$ & 3,5 & $0,0-9,2$ & 6,2 & 3,5 & 3,4 & 1,6 & 1,0 & 1,3 & $-43,5$ & $-2,9$ & $-45,2$ \\
\hline
\end{tabular}

Tabela 2

Número de óbitos por tuberculose, mortalidade proporcional por tuberculose em relação às doenças infecciosas e parasitárias (DIP) e variação anual segundo Distrito Sanitário de residência. Salvador, Bahia, Brasil, 1991, 1994 e 1997.

\begin{tabular}{|c|c|c|c|c|c|c|c|c|c|}
\hline \multirow[t]{2}{*}{ Distrito Sanitário } & \multicolumn{3}{|c|}{ Óbitos por tuberculose } & \multicolumn{3}{|c|}{$\begin{array}{l}\text { Mortalidade por tuberculose } \\
\text { em relação às DIP (\%) }\end{array}$} & \multicolumn{3}{|c|}{ Variação anual (\%) } \\
\hline & 1991 & 1994 & 1997 & 1991 & 1994 & 1997 & 1991-1994 & 1994-1997 & 1991-1997 \\
\hline I - Centro Histórico & 15 & 5 & 9 & 31,9 & 16,7 & 14,3 & $-47,6$ & $-14,4$ & $-55,2$ \\
\hline II - Itapagipe & 10 & 17 & 13 & 17,9 & 19,3 & 19,4 & 7,8 & 0,5 & 8,4 \\
\hline III - São Caetano/Valéria & 21 & 20 & 9 & 27,6 & 20,2 & 16,1 & $-26,8$ & $-20,3$ & $-41,7$ \\
\hline IV - Liberdade & 20 & 18 & 9 & 27,4 & 22,8 & 11,8 & $-16,8$ & $-48,2$ & $-56,9$ \\
\hline V-Brotas & 14 & 9 & 7 & 25,0 & 15,3 & 13,5 & $-38,8$ & $-11,8$ & $-46,0$ \\
\hline VI - Barra/Rio Vermelho/Pituba & 16 & 29 & 22 & 14,7 & 29,3 & 19,3 & 99,3 & $-34,1$ & 31,3 \\
\hline VII - Boca do Rio & 3 & 4 & 6 & 14,3 & 15,4 & 18,8 & 7,7 & 22,1 & 31,5 \\
\hline VIII - Itapuã & 10 & 10 & 6 & 20,8 & 20,4 & 12,2 & $-1,9$ & $-40,2$ & $-41,3$ \\
\hline IX - Cabula & 22 & 18 & 13 & 15,7 & 17,0 & 16,9 & 8,3 & $-0,6$ & 7,6 \\
\hline X - Pau da Lima & 9 & 5 & 12 & 12,9 & 5,6 & 21,8 & $-56,6$ & 289,3 & 69,0 \\
\hline XI - Subúrbio Ferroviário & 36 & 26 & 28 & 22,9 & 18,8 & 20,3 & $-17,9$ & 8,0 & $-11,4$ \\
\hline XII - Cajazeiras & 5 & 3 & 3 & 20,0 & 14,3 & 14,3 & $-28,5$ & 0,0 & $-28,5$ \\
\hline Salvador & 184 & 169 & 158 & 20,7 & 18,2 & 16,9 & $-12,1$ & $-7,1$ & $-18,4$ \\
\hline
\end{tabular}




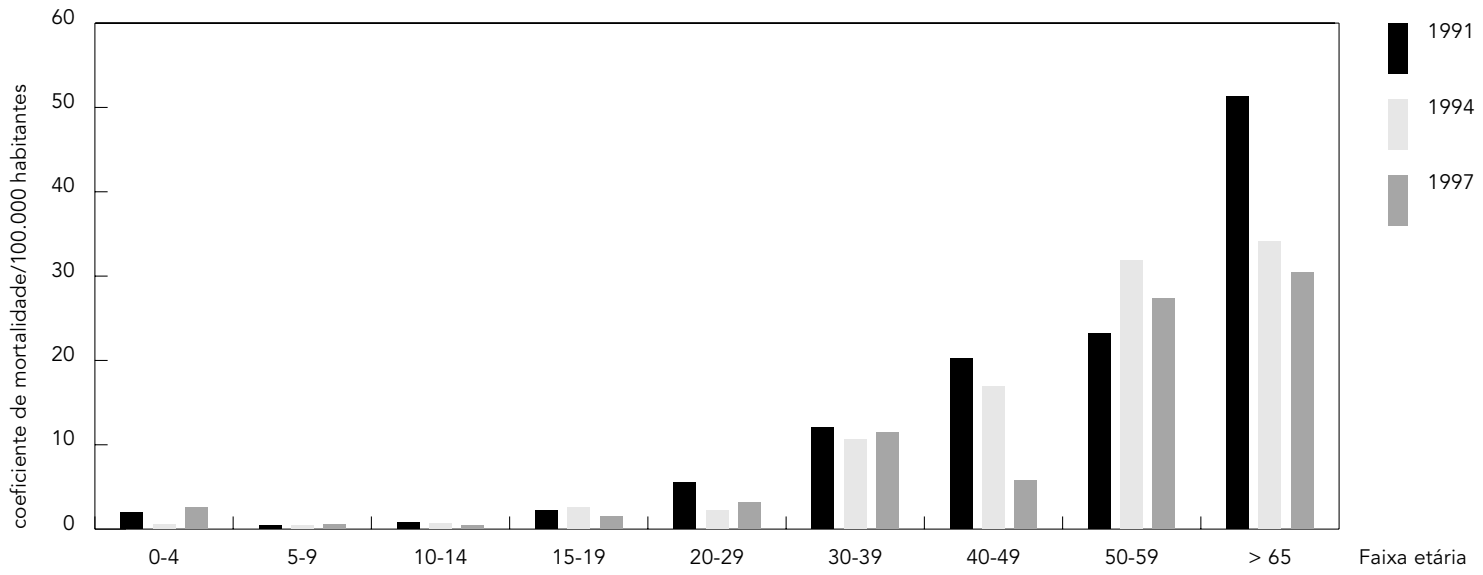

O achado de um maior risco de morte por tuberculose entre idosos (Figura 1) confirma os resultados de outros estudos (Antunes \& Waldman, 1999; Chalmowicz, 2001; Hijjar, 1992) Esse fato tem sido atribuído tanto a fatores demográficos relacionados ao envelhecimento da população, quanto à influência de programas de controle e da infecção por HIV (Chalmowicz, 2001) Com o envelhecimento da população, diversas pessoas que tiveram a primo infecção nas primeiras décadas do século XX, na terceira idade podem voltar a ficar susceptíveis. Por apresentarem quadros clínicos atípicos, essas pessoas não são facilmente diagnosticadas o que aumenta a mortalidade entre os idosos (Chalmowicz, 2001). Por outro lado, na medida em que a transmissão da tuberculose é reduzida, ocorre uma diminuição no risco de infecção e não no de reativação de lesões anteriores o que também contribui para o desvio para as faixas etárias mais elevadas (Chalmowicz, 2001).

A desigualdade da mortalidade por tuberculose entre os DS também foi muito acentuada (3 a 4 vezes em algumas situações). De modo geral, os DS que possuíam os coeficientes padronizados mais elevados abrangeram áreas situadas na periferia da cidade (Figura 2), onde grande parte das famílias habita em condições de vida precárias, o que possivelmente tornou os indivíduos mais expostos e mais susceptíveis a infecções oportunistas como a tuberculose. Esses elevados riscos de morte podem ainda estar revelando desigualdades e problemas relacionados com acesso aos serviços de saúde, a aderência ao tratamento ou na qualidade da atenção médica, na medida em que já se encontra demonstrada a efetividade dos tratamentos supervisionados, de curta duração (WHO, 2001a, 2001b). Assim, a identificação dos espaços de maior risco de óbito para essa doença, pode constituir-se em subsídio para a formulação de políticas capazes de melhorar o acesso e a qualidade dos serviços de saúde disponíveis a populações residentes nessas áreas.

A maior proporção de óbitos por tuberculose em relação às DIP observada em alguns DS, pode estar mais relacionada à magnitude do número de mortes por aquela causa do que a uma eventual diminuição dos óbitos devido a outras doenças infecciosas e parasitárias. Essa suposição baseia-se no fato de que apesar de o DS do Subúrbio Ferroviário ter tido o maior coeficiente padronizado em 1991, o segundo maior em 1994 e um número absoluto de óbitos bastante superior ao dos demais, esse DS situou-se apenas no quinto e no sexto lugares em relação à mortalidade proporcional, para os respectivos anos. Fica então evidente uma deficiência em relação ao controle e ao tratamento das DIP, incluindo aqui a tuberculose, no distrito em questão. 
Figura 2

Coeficiente padronizado de mortalidade por tuberculose segundo Distrito Sanitário.

Salvador, Bahia, Brasil, 1991, 1994 e 1997.
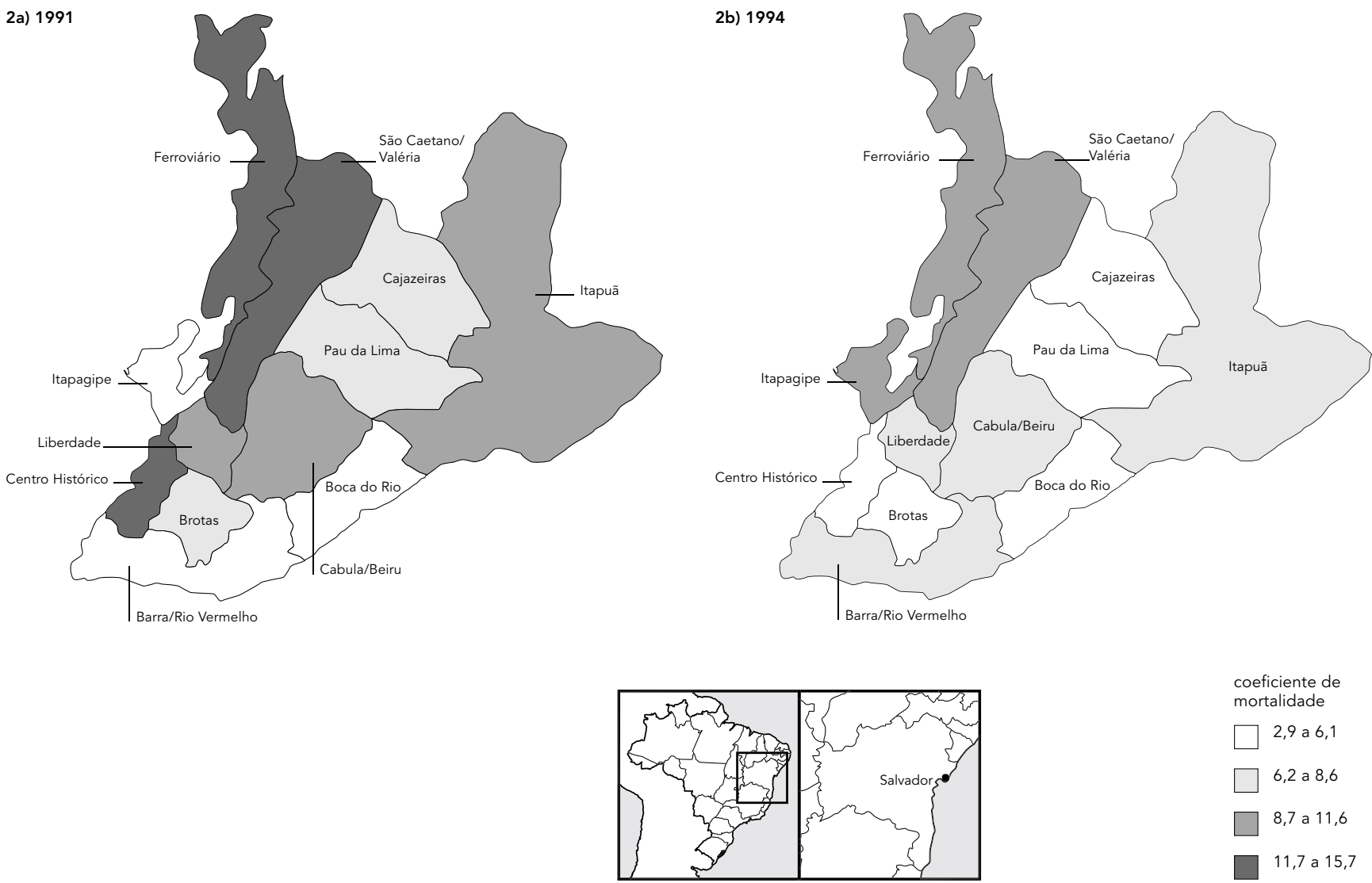

coeficiente de mortalidade

2,9 a 6,1

6,2 a 8,6

8,7 a 11,6

11,7 a 15,7

Embora não se possa analisar a tendência de evolução de um indicador de mortalidade a partir da descrição da situação do mesmo em apenas três anos, como foi feito no presente estudo, algumas hipóteses podem ser formuladas visando a sua exploração em investigações subseqüentes. Dessa forma, o crescimento da mortalidade nos DS Itapagipe (59,3\% e 47,5\%), Boca do Rio (26,3\% e 55,3\%) e Barra/Rio Vermelho/ Pituba (68,2\% e 27,3\%), respectivamente entre 1991 e 1994, e entre 1991 e 1997, tanto pode ter sido decorrente de problemas no programa de controle nesses distritos relacionados com cobertura, acesso e abandono quanto pode ter havido deslocamento de faixa etária ou mesmo melhoria no preenchimento dos atestados de óbito. Essa última hipótese é menos provável tendo em vista a reduzida proporção de óbitos por causas mal definidas em Salvador. Por outro lado, a redução observada nos demais distritos pode relacionar-se com melhorias na cobertura e qualidade das ações de controle decorrentes da descentralização das mesmas.

Analisar a situação da tuberculose a partir da mortalidade de forma isolada tem limitações. O coeficiente de mortalidade não tem sido considerado como um dos melhores indicadores para a vigilância da doença, devido ao fato de que a quimioterapia adequada reduz a taxa de letalidade a um nível extremamente baixo e no caso dos países periféricos a qualidade dos dados de mortalidade não é boa (Styblo, 1976). Contudo, no nosso meio, o controle ainda não conseguiu reduzir a mortalidade nos níveis observados nos países industrializados e a qualidade dos dados de mortalidade nas capitais 
tem permitido a realização de estudos. Além disso, em se tratando de óbito evitável, para o qual existem ações programáticas há três décadas, a persistência de taxas elevadas de mortalidade ao lado da sua distribuição desigual no espaço urbano revela limitações dos serviços de saúde no que diz respeito à cobertura, qualidade e eqüidade.

Os resultados aqui encontrados, de certa forma sugerem que o controle da tuberculose requer indiscutivelmente a melhoria progressiva das condições de vida da população da cidade, apontando também, para a necessidade de implementação de políticas de saúde, voltadas para populações residentes em áreas de maior risco em relação à morte por tuberculose em Salvador, no sentido de progredir no controle do problema no município.

De fundamental importância é a adoção de medidas intersetoriais de promoção e vigilância da saúde, capazes de atuar nos fatores condicionantes de forma articulada, que não apenas monitore casos, surtos e comunicantes, mas também alie a estratégia DOTS visando eliminar, principalmente, a baixa aderência ao tratamento e o surgimento de cepas resistentes (WHO, 2001b).

O MS, por meio de seu último Plano Nacional de Controle da Tuberculose, pretende abranger a totalidade dos municípios brasileiros, inclusive com a implantação do tratamento supervisionado (MS, 1999). Cabe à Secretaria Municipal de Saúde (SMS), com o apoio da SESAB, participar ativamente desse esforço para reduzir essas mortes evitáveis na cidade de Salvador.

\section{Agradecimentos}

A Alcione Brasileiro Oliveira Cunha pela contribuição na elaboração das tabelas e mapas referentes ao ano de 1997.
Figura 2 (continuação)

Coeficiente padronizado de mortalidade por tuberculose segundo Distrito Sanitário. Salvador, Bahia, Brasil, 1991, 1994 e 1997.
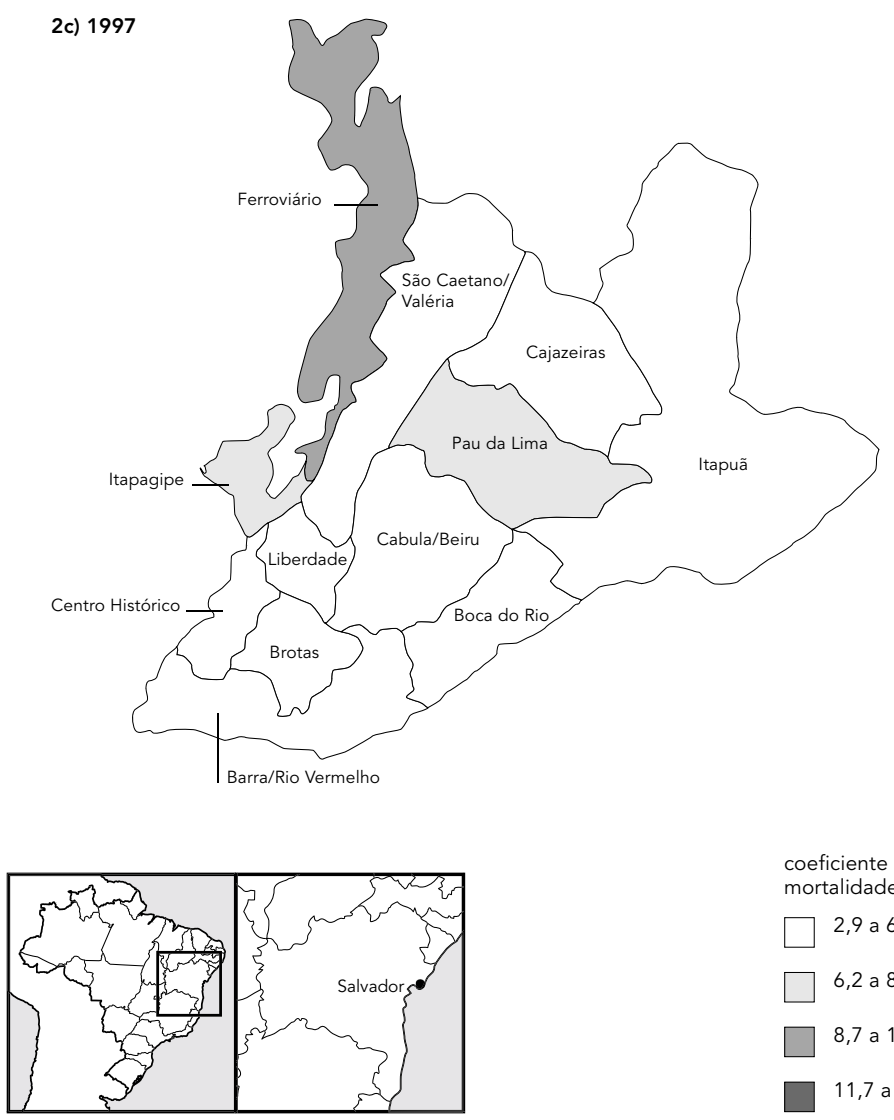

coeficiente de mortalidade

2,9 a 6,1

6,2 a 8,6

8,7 a 11,6

11,7 a 15,7

\section{Referências}

ANTUNES, J. L. F. \& WALDMAN, E. A., 1999. Tuberculosis in the twentieth century: Time-series mortality in São Paulo, Brazil, 1900-97. Cadernos de Saúde Pública, 15:463-476.

MS (Ministério da Saúde), 1999. Manual de Normas para o Controle da Tuberculose. Brasília: MS.

CARNEIRO, N. M. B. \& MOTA, E., 1986. Tuberculose em Salvador, Bahia: Incidência e algumas variáveis sociodemográficas em 1980. Revista Baiana de Saúde Pública, 13:68-80.

CHALMOWICZ, F., 2001. Transição etária da incidência e mortalidade por tuberculose no Brasil. Revista de Saúde Pública, 35:81-87.

DYE, C.; WATT, C. J. \& BLEED, D., 2002. Low access to a highly effective therapy: A challenge for international tuberculosis control. Bulletin of the World Health Organization, 80:437-444. 
HIJJAR, M. A., 1992. Epidemiologia da tuberculose no Brasil. Informe Epidemiológico do SUS, 6:1-69.

HOPEWELL, P. C., 2002. Tuberculosis control: How the world has changed since 1990. Bulletin of the World Health Organization, 80:427-427.

LEE, J. W.; LOEVINSOHN, E. \& KUMARESAN, J. A., 2002. Response to a major disease of poverty: The Global Partnership to Stop TB. Bulletin of the World Health Organization, 80:428-428.

LIMA, N. M. C. C., 1983. Tuberculose em Salvador: Distribuição Espacial da Doença. Dissertação de Mestrado, Salvador: Universidade Federal da Bahia.

MENEZES, M. L. G., 1986. A Recidiva de Tuberculose em Salvador, Bahia: Estudo das características dos Casos de Retratamento Notificados entre 1979 e 1984. Dissertação de Mestrado, Salvador: Universidade Federal da Bahia.

RAVIGLIONE, M. C.; SNIDER, D. E. \& KOCHI, A., 1996. Global epidemiology of tuberculosis. Morbidity and mortality of a worldwide epidemic. JAMA, 273:220-226.

ROTHMAN, K. J., 1986. Modern Epidemiology. Boston/ Toronto: Little Brown and Co.

RUFFINO-NETTO, A., 2002. Tuberculose: A calamidade negligenciada. Revista da Sociedade Brasileira de Medicina Tropical, 35:51-58.
RUFFINO-NETTO, A. \& SOUZA, A. M. A. F., 1999. Reforma do setor saúde e controle da tuberculose no Brasil. Informe Epidemiológico do SUS, 8:35-51.

STYBLO, K., 1976. Surveillance of tuberculosis. International Journal of Epidemiology, 5:63-68.

WHO (World Health Organization), 1999. What is DOTS? A Guide to Understanding the WHO-Recommended TB Control Strategy Known as DOTS. Geneva: WHO.

WHO (World Health Organization), 2001. World TB day 2001: Access to TB cure a Human Rights Imperative. April $2001<\mathrm{http}$ ///www.who.int/inf-pr-200114.html>.

WHO (World Health Organization), 2001. Global Tuberculosis Control, 2001. WHO Report 2001. Geneva: WHO.

WHO (World Health Organization), 2002. Global Tuberculosis Control. Surveillance, Planning, Financing. WHO Report 2002. 22 June 2002 <http:/ / www. who.int/gtb/publications/globrep02/index.html>.

Recebido em 16 de junho de 2001

Versão final reapresentada em 21 de outubro de 2002 Aprovado em 10 de dezembro de 2002 